\title{
INTERPRETIVE SUMMARIES, FEBRUARY 2010
}

Invited review: Technical solutions for analysis of milk constituents and abnormal milk. By Brandt et al., page $42 \%$. The knowledge of alterations in milk constituents can be suitable for management support in terms of mastitis detection, feeding, and reproduction management in dairy farms. Numerous sensors for this purpose are either available or in development. This review article describes potential techniques for on-farm or on-line detection of milk constituents, their typical applications, and their usefulness.

Investigation of the microstructure of milk protein concentrate powders during rehydration: Alterations during storage. By Mimouni et al., page 463. Scanning electron microscopy was used to investigate the microstructure of milk protein concentrate powder. The dissolution process and the effects of prior storage of the powder were characterized. Different types of interactions (bridges, direct contact) between casein micelles resulted in a porous, gel-like structure that restrained the dispersion of individual micelles into the surrounding liquid phase. During storage of the powder, increased interactions occured between micelles, leading to compaction of micelles and the formation of a monolayer skin of casein micelles packed close together, the combination of which are proposed to be responsible for the slow dissolution of stored powders.

Blocking of Pseudomonas aeruginosa and Chromobacterium violaceum lectins by diverse mammalian milks. By Zinger-Yosovich et al., page 473. Milk protection of newborns against infections includes blocking the lectins (sugar-binding proteins) that anchor pathogens to infants' cells. Use of 3 lectins from Pseudomonas aeruginosa (PA-IL and PA-IIL) and Chromobacterium violaceum (CV-IIL) as probes for 13 milks demonstrated that human, alpaca, giraffe, and monkey milks were the best inhibitors. Mare, buffalo, rabbit, and camel milks inhibited either PA-IL or PAIIL and CV-IIL. Cow, deer, dog, goat, and sheep milks were weak inhibitors. The efficient milk glycans might be used for preventing skin, ear, eye, and gut infections by lectin-mediated adhesion of the above and similar pathogens

Dynamic high pressure-induced gelation in milk protein model systems. By Venir et al., page 483. Improvement of milk protein functionality by means of high pressure homogenization is of great interest in the dairy field. The aim of the current work was to evaluate the effect of different homogenization pressures on gelation and thickening properties of both single and mixed sodium caseinate-whey protein concentrate model systems. By setting appropriate process conditions and concentration of milk protein dispersions, systems or gels with tailored characteristics may be obtained. Dynamic high pressure treatment after heating led to stronger gels.

Modification to the renneting functionality of casein micelles caused by nonionic surfactants. By Ion Titapiccolo et al., page 506. When Tween is added to milk, the rennet-induced aggregation of the casein micelles is affected. The presence of Tween accelerates both the first and second phase of renneting in skim milk. The gel obtained shows a higher elastic modulus than that of a skim milk gel, but with similar brittleness. By varying the size of the surfactant (Tween 20 or Tween 80) as well as the colloidal state of the proteins in solution, it was possible to demonstrate that the surfactant does not have a direct effect on the chymosin activity, but rather on the casein micelles. It was also demonstrated that the effect of surfactant on the gelation point is reduced by increasing surfactant size. These findings can improve our ability to customdesign final structures in dairy products.

Measurement of ionic calcium, $\mathrm{pH}$, and soluble divalent cations in milk at high temperature. By On-Nom et al., page 515. Milk was partitioned by ultrafiltration and dialysis at different temperatures. Ionic calcium and $\mathrm{pH}$ of dialysates and permeates both decreased as temperature increased. There was good agreement between results from dialysis and ultrafiltration over the temperature range 20 to $80^{\circ} \mathrm{C}$, and dialysis provided reproducible results from 90 to $120^{\circ} \mathrm{C}$. Ionic calcium and $\mathrm{pH}$ of permeates and dialysates collected at high temperature but measured at $20^{\circ} \mathrm{C}$ were much lower than values found for heated milk after it had cooled to $20^{\circ} \mathrm{C}$. Measuring $\mathrm{pH}$ and ionic calcium at high temperatures will facilitate a better understanding of their roles in heat stability of milk.

Short communication: The effect of dry period duration and dietary energy density in early lactation on the rennet gelation properties of milk. By Butler et al., page 524. The rennet gelation characteristics of milk samples collected at 2, 6, and 10 wk postpartum were compared in cows given 1 of 2 planned dry period lengths ( 0 or $8 \mathrm{wk}$ ) and 1 of 2 feeding levels (standard or high-energy TMR). Decreasing dry period duration resulted in higher postpartum milk protein concentrations and was associated with greater maximum curd firming rate and gel strength of milk following rennet addition. Feeding level had no effect on milk protein concentration or rennet gelation characteristics. Decreasing dry period duration may have beneficial effects on the processability of milk in the subsequent lactation. 
Effects of dietary fats differing in n-6:n-3 ratio fed to high-yielding dairy cows on fatty acid composition of ovarian compartments, follicular status, and oocyte quality. By Zachut et al., page 529. The effects of dietary fats differing in n-6:n-3 ratio on fatty acid composition of ovarian compartments and on follicular status, preovulatory follicles, and oocyte quality were examined. Cows were fed either a control diet or a diet supplemented with encapsulated flax or sunflower seed oil. Flaxseed increased the proportion of C18:3 (n-3) in milk, plasma, follicular fluid, granulosa cells, and cumulus-oocyte complexes. Differences were observed in follicular status, and oocyte cleavage rate was higher in the flaxseed cows than in controls.

Evaluation of nonesterified fatty acids and $\beta$-hydroxybutyrate in transition dairy cattle in the northeastern United States: Critical thresholds for prediction of clinical diseases. By Ospina et al., page 546. Associations between prepartum nonesterified fatty acids (NEFA), postpartum $\beta$-hydroxybutyrate, and postpartum NEFA serum concentrations and clinical diseases were evaluated using a data set of 2,758 cows from 100 herds. Elevated serum $\beta$-hydroxybutyrate postpartum and NEFA pre- and postpartum all were associated with the development of metritis, clinical ketosis, and displaced abomasa; however, among these risk factors, postpartum NEFA concentrations were most associated with the development of clinical disease.

Effect of milking frequency on lactation persistency and mammary gland remodeling in midlactation cows. By Bernier-Dodier et al., page 555 . Mid-lactation cows were milked differentially for $8 \mathrm{wk}$, with half of the mammary gland milked once daily and the other half milked thrice daily. Milk production and lactation persistency were greater in the gland pair that was milked more frequently. Differential milking also had multiple effects on milk composition and on the mammary cells, including the modulation of proliferation, apoptosis, and gene expression. This study presents information on events that could be implicated in the modulation of milk yield and lactation persistency.

Effects of increased milking frequency on metabolism and mammary cell proliferation in Holstein dairy cows. By Soberon et al., page 565. Holstein cows were subjected to increased milking frequency (IMF) for the first $21 \mathrm{~d}$ of lactation, followed by a return to twice-daily milking. Production responses to IMF were more pronounced during the period of IMF, and carryover responses were minimal. Overall, primiparous cows had greater milk yield responses to IMF than did multiparous cows. Increased concentrations of blood markers for energy balance suggested greater body fat mobilization in multiparous cows subjected to
IMF. Results from mammary biopsies suggested that IMF did not affect mammary cell proliferation and that effects of IMF must be mediated through other mechanisms.

The effect of treatment with long-acting antibiotic at postweaning movement on respiratory disease and on growth in commercial dairy calves. By Stanton et al., page 574. Bovine respiratory disease is a major concern for replacement heifer raisers. This disease has severe economic consequences and is a welfare concern. The use of a single injection of tulathromycin, a long-acting antibiotic, at the time replacement calves were moved from individual to group housing decreased the incidence of respiratory disease and minimized the associated decrease in growth.

Quantifying degree of mastitis from common trends in a panel of indicators for mastitis in dairy cows. By Højsgaard and Friggens, page 582. Categorizing mastitis as either clinically sick or healthy is no longer sufficient, especially with today's on-farm measuring technology. We show that a quantitative degree of mastitis measure can be derived from a panel of indicators such as somatic cell count, conductivity, and a mastitis enzyme (all automatically measurable on-farm). Further, we show that this degree of infection measure detects intramammary infection up to $5 \mathrm{~d}$ prior to clinical signs and treatments for mastitis. This method has general applicability to other diseases.

Proteomic analysis of the temporal expression of bovine milk proteins during coliform mastitis and label-free relative quantification. By Boehmer et al., page 593. Temporal expression of abundant milk proteins in milk from healthy cows and cows with experimentally induced coliform mastitis was analyzed using liquid chromatography coupled with tandem mass spectrometry (LC-MS/MS). A label-free approach was used to quantify relative changes in identified proteins, and observed trends in protein modulation detected using LC-MS/MS were compared with changes in protein concentration measured using an ELISA. The goals of the study were to investigate the feasibility of using a non-antibody-based approach to track changes in biomarker expression during mastitis. Results indicate that label-free LC-MS/MS methods are a viable means of tracking changes in relative protein abundance in milk during mastitis.

Evaluation of two communication strategies to improve udder health management. By Jansen et al., page 604. This study evaluates two communication strategies used in a mastitis control program in the Netherlands: the central route, by distribution of science-based educational tools such as a handbook on 
udder health, and the peripheral route, which employs a mass media campaign without rational argumentation to promote the use of milking gloves. Depending on farmers' motivation and aim of the campaign, both strategies are effective in reaching their target audience. However, combining the two strategies would maximize a campaign's goal to reach as many dairy farmers as possible.

Reproductive risk factors for culling and productive life in large dairy herds in the eastern United States between 2001 and 2006. By De Vries et al., page 613. Survival and reproductive risk factors for culling in Holstein dairy herds with at least 200 cows were described. The risk of culling increased with parity number and, for open cows, after $280 \mathrm{~d}$ after calving. Cows that had greater calving difficulty, gave birth to males or twins, were in herds with shorter days to first insemination, or had longer days to conception had increased risks of culling. The risk of culling was slightly greater for cows in herds where a synchronized breeding program was used. Knowledge of risk factors for culling is useful when making insemination and culling decisions.

Short communication: Repeatability of measures of rectal temperature in dairy cows. By Burfeind et al., page 624. The objective was to evaluate variability of rectal temperatures in dairy cows considering different factors (intra- and interinvestigator repeatability, different thermometers, penetration depth into the rectum, and defecation). High coefficients of correlation and small differences between values of rectal temperatures provide evidence that rectal temperature was a repeatable measure in dairy cows, according to the factors described. Measurements can be influenced by the procedure of obtaining the temperature, the type of thermometer, and penetration depth into the rectum. Differences in rectal temperature before and after defecation were minor.

Conservation characteristics of wilted perennial ryegrass silage made using biological or chemical additives. By Conaghan et al., page 628. The amount of sugar in silage fed to dairy cows can be an important factor influencing nitrogen excretion in urine. An increase in the sugar content of silage should reduce the quantity of nitrogen excreted, potentially increasing milk production and reducing environmental pollution. This study evaluated the ability of 7 different additive treatments, including biological and chemical additives, to increase the sugar content of grass silage at feeding. We found significant differences among additives in silage quality. However, none of the additive treatments evaluated could consistently increase silage sugar content. The strengths and weaknesses of the additives were discussed.
Effects of heat stress on energetic metabolism in lactating Holstein cows. By Wheelock et al., page 644. Heat stress-induced decrease in feed intake accounted for approximately $50 \%$ of the decrease in milk yield. Heat-stressed cows have increased basal and stimulated insulin, which markedly alters postabsorptive carbohydrate and lipid metabolism. This is primarily characterized by an increase in glucose disposal following a glucose tolerance test and prevention of adipose tissue lipid mobilization, although calculated bioenergetics and body weight loss would predict the opposite. It appears that the heat-stressed lactating cow cannot employ typical glucose-sparing mechanisms and, thus, milk yield is decreased more than would be predicted based upon nutrient intake.

High dietary iron reduces transporters involved in iron and manganese metabolism and increases intestinal permeability in calves. By Hansen et al., page 656. Cattle diets are often high in iron, which may have negative effects on the metabolism of manganese. Our work suggests that high dietary iron decreases gain and efficiency of gain in calves. We also report that proteins involved in the cellular uptake and export of iron in the intestine are decreased when young calves are fed a diet high in iron. At least one of these proteins, the iron import protein divalent metal transporter 1, has been shown in other species to be important for manganese absorption. In our study, we found that manganese accumulation in the intestine was decreased when calves were fed high dietary iron, suggesting that decreases in divalent metal transporter 1 at the gene and protein levels may have contributed to reduced absorption of manganese.

The effects of forage proportion and rapidly degradable dry matter from concentrate on ruminal digestion in dairy cows fed corn silagebased diets with fixed neutral detergent fiber and starch contents. By Lechartier and Peyraud et al., page 666. Modern farms aim to maximize milk production with high-energy diets, which can trigger ruminal acidosis. This trial studied the effects of forage proportion and concentrate ruminal degradation rate with fixed dietary neutral detergent fiber and starch contents. Rumen $\mathrm{pH}$ decreased when decreasing the proportion of fiber from forage and increasing the concentrate degradation rate, which was explained by a reduced chewing time and an accelerated fermentation rate, respectively. Prediction of ruminal $\mathrm{pH}$ is improved when considering both the proportion of fiber from forage and the concentrate degradation rate.

Effect of Saccharomyces cerevisiae fermentation product on ruminal fermentation and nutrient utilization in dairy cows. By Hristov et al., page 682. This experiment investigated the effects of yeast culture 
on ruminal fermentation and nitrogen losses in dairy cows. The yeast culture tended to decrease ruminal ammonia concentration, tended to increase microbial protein synthesis in the rumen, and reduced ammonia and methane emissions from manure. Production of the cows and milk composition were not affected.

Effects of nonstructural carbohydrate concentration in alfalfa on fermentation and microbial protein synthesis in continuous culture. By Berthiaume et al., page 693. Strategies to improve N utilization in dairy cows are required to reduce the environmental effect of farms. We hypothesized that selecting alfalfa with a higher concentration of nonstructural carbohydrates would balance the supply of energy and protein in the rumen, leading to an increase in bacterial protein synthesis. Feeding alfalfa with a higher NSC concentration promoted a more glucogenic fermentation and enhanced microbial- $\mathrm{N}$ synthesis in the rumen.

Effects of fumarate on ruminal ammonia accumulation and fiber digestion in vitro and nutrient utilization in dairy does. By Yu et al., page 701. Organic acid fumarate is a key metabolic intermediate for rumen microorganisms. Addition of fumarate to rumen microbial incubation enhanced utilization of ammonia and digestion of feed fiber. When fumarate was supplemented to dairy does, feed consumption was unaffected. However, digestion of diet gross energy, crude protein, and cellulose was improved and concentration of plasma glucose was increased. Fumarate benefited rumen microbial fermentation and animal performance.

Effect of feeding a reduced-starch diet with or without amylase addition on lactation performance in dairy cows. By Gencoglu et al., page 723. High corn prices have heightened the interest in reduced-starch diets. Some exogenous dietary enzymes may improve animal performance. The objective of this trial was to determine lactation performance responses in high-producing dairy cows to a reduced-starch diet and to addition of exogenous amylase. Feed to milk conversions $(\mathrm{kg} / \mathrm{kg}$ of DMI) were 12 to $13 \%$ greater for cows fed a reduced-starch diet with exogenous amylase added, which may improve economic performance.

Hot topic: A unified approach to utilize phenotypic, full pedigree, and genomic information for genetic evaluation of Holstein final score. By Aguilar et al., page 743. The first national, singlestep, full-information (phenotype, pedigree, and marker genotype) genetic evaluation was developed for final score of United States Holsteins. Single-step evaluations were as accurate as multiple-step evaluations and more widely applicable. The methodology is valid for any species or data structure.
Diacylglycerol acyltransferase 1, stearoyl-CoA desaturase 1, and sterol regulatory element binding protein 1 gene polymorphisms and milk fatty acid composition in Italian Brown cattle. By Conte et al., page 753. Relationships among stearoyl-CoA desaturase 1 (SCD1), diacylglycerol acyltransferase 1 (DGAT1), and sterol regulatory element binding protein 1 (SREBP-1) gene polymorphisms and milk fatty acid content of 351 Italian Brown cows were investigated. Significant associations between stearoylCoA desaturase 1 and diacylglycerol acyltransferase 1 polymorphisms and cis-9 unsaturated:saturated fatty acid ratios, which represent a proxy of mammary desaturase activity, were revealed. A significant association between the above-mentioned genes and myristoleic:myristic ratio was also discovered. In contrast, sterol regulatory element binding protein 1 polymorphism did not seem significantly associated with fatty acid composition.

Comparative grazing behavior of lactating Holstein-Friesian, Jersey, and Jersey $\times$ HolsteinFriesian dairy cows and its association with intake capacity and production efficiency. $B y$ Prendiville et al., page 764. This is the first study to provide insight into the comparative grazing behavior of Holstein-Friesian, Jersey, and Jersey $\times$ HolsteinFriesian cows under grazing conditions. The study illustrates that fundamental differences in grazing and ruminating behavior do exist between the breeds. Evidence is presented to suggest that the crossbreed may display hybrid vigor for grazing efficiency, supporting the theory that Jersey $\times$ Holstein-Friesian cows are well suited to intensive grazing systems. The results obtained also indicate that inherent grazing and ruminating differences exist between cows varying in intake capacity and production efficiency.

The spatial expression pattern of antimicrobial peptides across the healthy bovine udder. By Tetens et al., page 775. This study describes a gene expression analysis of 6 antimicrobial peptides in different localizations of the healthy bovine mammary gland in order to further elucidate their role in udder health. Antimicrobial peptides are effector molecules of innate immunity displaying direct antimicrobial activity, thus being important for the resistance to mastitis. The identification of genomic variation resulting in individual expression differences might substantially contribute to the genetic improvement of udder health.

Short communication: Molecular genetic characterization of ovine $\alpha_{\mathrm{S} 1}$-casein allele $\mathrm{H}$ caused by alternative splicing. By Giambra et al., page 792. Ovine $\alpha_{S 1}$-casein allele $H$ previously demonstrated in East Friesian dairy sheep by isoelectric focusing was characterized at the molecular level. The variant is 
caused by skipping of exon 8 in pre-mRNA processing, resulting in an abbreviated protein. Sequence differences deleting the 5 '-donor splice site of intron 8 were detected at DNA level and determined as reason for alternative splicing. The shortening of $\mathrm{CSN}_{1 S}{ }^{*} \mathrm{H}$ mRNA sequence may affect the specific casein expression and characteristics of the protein.

Short communication: Characterization of a monoclonal antibody for $\kappa$-casein B of cow's milk. By Summer et al., page 796. On milk proteins, $\kappa$-casein is the most interesting and important component in that it is centrally involved in the coagulation of milk by rennet and in many other technologically important properties of the milk protein system. There are several genetic variants of $\kappa$-casein. The most diffused variants in cattle breeds are $\mathrm{A}$ and $\mathrm{B}$. The $\mathrm{B}$ variant in milk is associated with better coagulation properties and higher cheese yield than the A variant. In this study, a monoclonal antibody specific for this variant was characterized.

Evaluation of increased vitamin D fortification in high-temperature, short-time-processed $2 \%$ milk, UHT-processed $2 \%$ fat chocolate milk, and low-fat strawberry yogurt. By Hanson and Metzger, page 801. An increased level of vitamin $\mathrm{D}$ fortification in high-temperature, short-time-pasteurized $2 \%$ fat milk, UHT-pasteurized $2 \%$ fat chocolate milk, and lowfat strawberry yogurt was studied over the duration of each product's respective shelf life. Increased vitamin D fortification was stable over all product shelf lives and did not have an effect on the sensory characteristic of the products. These results indicate that it is feasible to increase vitamin D fortification to $250 \mathrm{IU}$ per serving in these products.

Recommendations for rationalizing cleaning-in place in the dairy industry: Case study of an ultra-high-temperature heat exchanger. By Alvarez et al., page 808. Cleaning-in-place systems are commonly used in the food industry for ensuring hygienic safety of foods and for recovering plant performance. This work proposes a new strategy based on objective and rational arguments and calculations in order to reduce the operating time and the volume and load of effluents produced during the cleaning. The strategy was validated for each phase of the sequences used for the cleaning of an industrial sterilizer. As a result, the overall duration of cleaning sequence and wastewaters volume were reduced by half, and significant reductions of detergent volume were achievable.

Feeding management and characteristics of rations for high-producing dairy cows in freestall herds. By Endres and Espejo, page 822. Observational data was collected from 50 randomly selected dairy farms in Minnesota. The majority of these freestall dairies fed cows once daily in 3-row pens with post and rail feed barriers and allowed $0.45 \mathrm{~m}$ of feed bunk space per cow. The most commonly used forage in the ration was corn silage, and all rations contained adequate amounts of neutral detergent fiber and crude protein. Ration neutral detergent fiber content changed over time, and this change was associated with some feeding management and total mixed ration characteristics that could be modified to achieve better feed utilization and productivity in dairy cows. 